\title{
Acute medullar compression secondary to a septic arthritis of a thoracic facet joint: a case report and review of literature
}

\author{
Sandra Huguet ${ }^{1} \cdot$ Natàlia Ibáñez $^{2} \cdot$ Martí Bernaus $^{3} \cdot$ Lluís Font-Vizcarra $^{4}$
}

Received: 8 July 2018 / Revised: 9 August 2018 / Accepted: 12 August 2018

(c) International Spinal Cord Society 2018

\begin{abstract}
Introduction Septic arthritis of a facet joint (SAFJ) is a rare entity and frequently underdiagnosed. It is most frequently localised in the lumbar segment.

Case presentation A 48-year-old woman presented at our emergency room with a chief complaint of lumbar pain and perineal hypoesthesia. An urgent lumbar Magnetic Resonance Imaging (MRI) was performed showing no signs of compressive pathology. Intravenous painkillers were administered improving her clinical symptoms, so she was discharged from the emergency department to be followed at our out-patient clinic. After $12 \mathrm{~h}$, the patient presented again referring an acute loss of strength in her lower limbs and paresthesias at a T10 sensitive level. Due to the severity of the symptoms, we performed an urgent dorsal MRI that showed a septic arthritis of the left T8-T9 facet joint and an epidural abscess causing a medullar compression. Blood cultures were performed before surgical treatment. An urgent laminectomy of T8 and debridement of the abscess were performed. Intraoperative microbiology and blood cultures were positive for Streptococcus constellatus. After surgery, the patient presented a progressive improvement of her symptomatology.

Discussion Physicians should have in mind and rule out this clinical entity to avoid severe consequences that a misdiagnosed SAFJ could develop.
\end{abstract}

\section{Introduction}

Septic arthritis of a facet joint (SAFJ) is a rare medical entity. Only a few cases have been described in the literature since Halpin's publication in 1987 [1].

The incidence of SAFJ shows variations according to different reviews and is estimated to be about 4\% [2] to $20 \%$ [3] of all spine infections. These incidence rates are far below spondylodiscitis. Most cases relate to an infection by

Sandra Huguet

sanhumi@gmail.com

1 Department of Traumatology and Orthopedics, Hospital Universitari Mútua de Terrassa, Barcelona, Spain

2 Department of Traumatology and Orthopedics, Consorci Sanitari del Garraf, Barcelona, Spain

3 Osteoarticular Infections Unit - Department of Traumatology and Orthopedics, Hospital Universitari Mútua de Terrassa, Barcelona, Spain

4 Osteoarticular Infections Unit - Department of Traumatology and Orthopedics, Hospital Universitari Mútua de Terrassa, Barcelona, Spain haematogenous spread, although entry portal remains unknown for one third of the patients [4].

Some previously described risk factors $[2,5]$ are diabetes mellitus, concurrent extraspinal infection, chronic corticosteroid treatment or epidural injection.

SAFJ is most frequently localised in the lumbar segment $(86-97 \%)[3,5,6]$. Many fewer cases are described for the cervical or thoracic vertebrae.

SAFJ is frequently underdiagnosed. It can begin with very mild symptoms and could be very similar to spondylodiscitis. According to the literature, about 1 month is the average delay between symptoms and diagnosis of SAFJ [2, $3,6,7]$. Fever is present at the beginning of symptoms in $60-80 \%$ of the cases $[2,3,7]$. Due to the seriousness of the consequences of a delayed diagnosis and treatment of SAFJ, it becomes very important to include this entity in the differential diagnosis for back pain with or without fever.

\section{Case presentation}

We report a 48-year-old woman who presented at our emergency room with a chief complaint of lumbar pain and perineal hypoesthesia. 


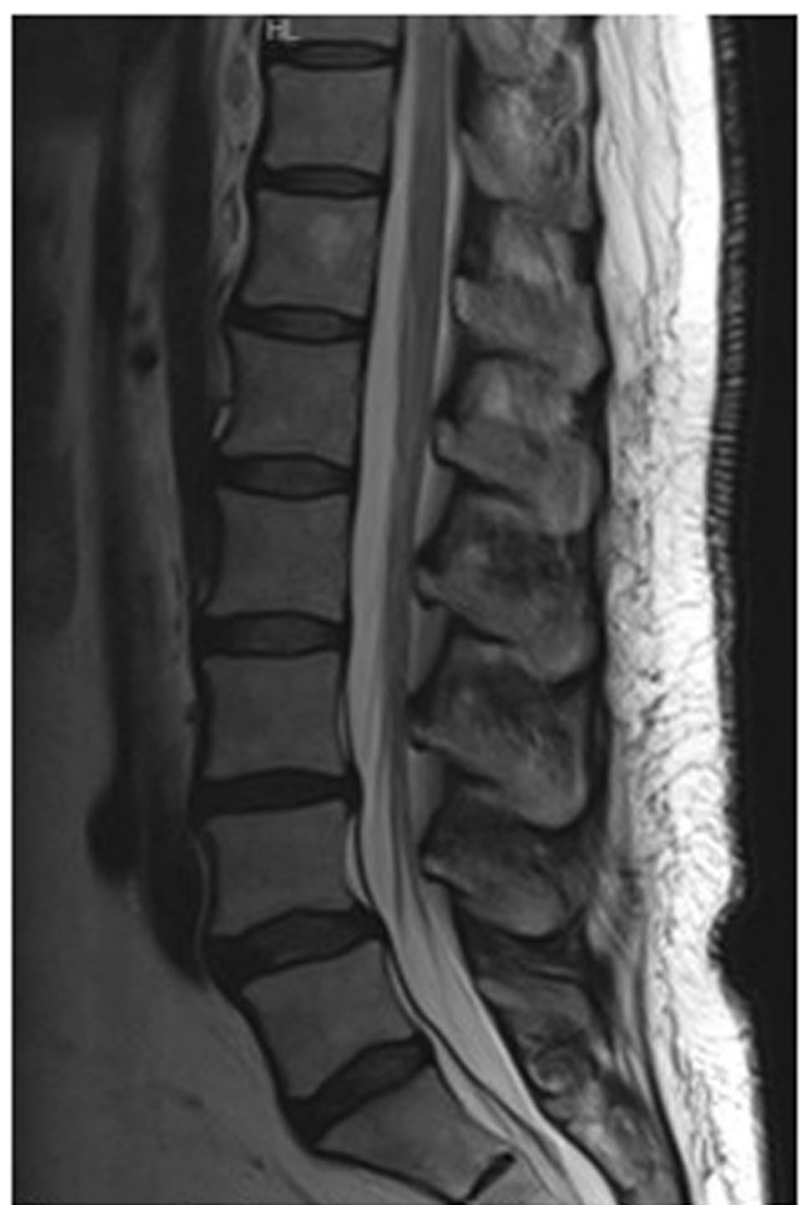

Fig. 1 Lumbar MRI without any compressive pathology

Medical history included diabetes mellitus, hypertension and obesity.

Physical examination did not show any motor deficits or sphincter alterations.

Due to her sensitive symptoms we decided to perform an urgent lumbar MRI and intravenous analgesia was administered. The MRI showed mild L4-L5 anterolisthesis and L3-S1 discal degeneration but no signs of any compressive pathology (Fig. 1).

After observation and improvement of symptomatology with intravenous analgesia, the patient was discharged from our emergency department to be followed at our out-patient clinic.

After $12 \mathrm{~h}$, the patient presented again referring an acute loss of strength in her lower limbs and paresthesias at a T10 sensitive level.

Physical examination showed no fever, a remarkable motor deficit of the left psoas and quadriceps scoring $1 / 5$ (according to the Oxford Scale for Muscle Strength), a T10 level hypoesthesia, hypotonic anal sphincter and perineal anaesthesia without incontinence. All reflexes of the left

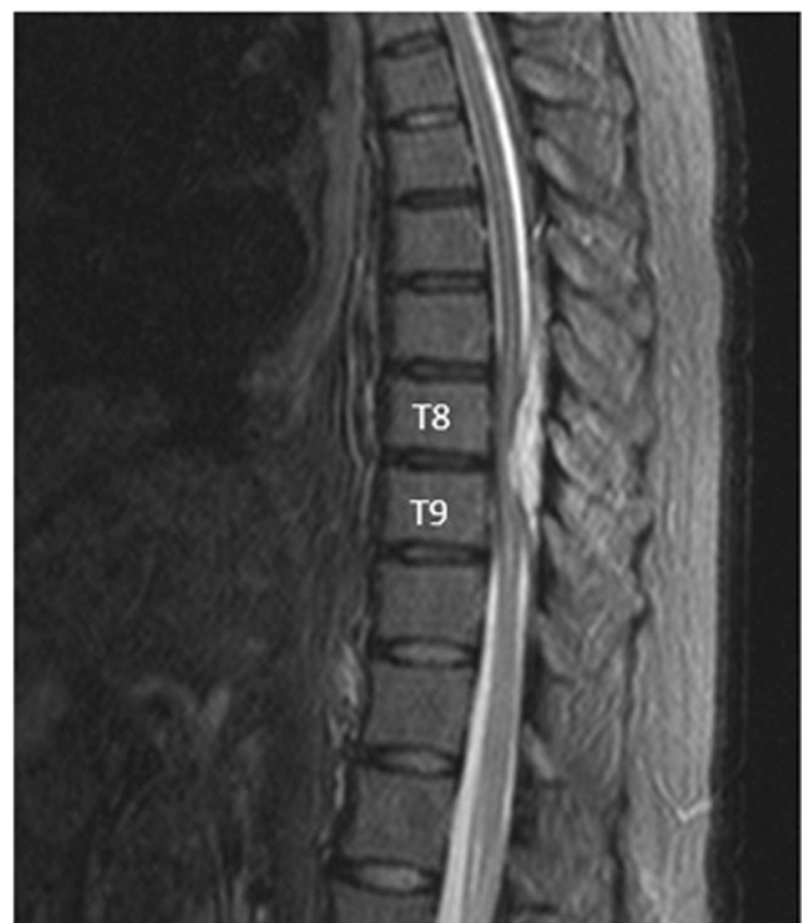

Fig. 2 Dorsal MRI shows an epidural abscess $(34 \times 10 \times 11 \mathrm{~mm})$. $\mathrm{T} 2$ sequence.

inferior limb were decreased with a presence of a positive Babinski.

No alterations were observed in cervical, dorsal and lumbar plain radiographs.

Blood test showed leukocytosis with a mild left deviation (total white blood-cell count was $10.82 \times 10^{9} / \mathrm{L}$ with $78.6 \%$ neutrophils), C-reactive protein (CRP) $79.1 \mathrm{mg} / \mathrm{dL}$ and erythrocyte sedimentation rate (ESR) $43 \mathrm{~mm}$. Blood cultures were performed.

We diagnosed our patient as an incomplete medullar lesion type ASIA D. An urgent dorsal MRI was performed showing an image compatible with a septic arthritis of the T8-9 left facet joint and an epidural abscess $(34 \times 10 \times$ $11 \mathrm{~mm}$ ) causing a medullar compression with signs of myelopathy (Figs. 2, 3, 4 and 5).

An urgent debridement and laminectomy of T8 were performed. Abundant purulent material was found connecting the facet joint and the abscess. Severe bone destruction along with disappearance of the capsule of the T8-9 left facet joint was seen. During the surgery, several microbiological and histological samples were taken.

In the immediate post-operative period, and according to the protocol of our hospital, empiric antibiotic therapy was initiated with Ceftriaxone $2 \mathrm{~g} / 24 \mathrm{~h}$ and Cloxacillin $2 \mathrm{~g} / 24 \mathrm{~h}$.

Two days after surgery, blood (1/2) and intraoperative tissue cultures (6/6) were positive for Streptococcus constellatus. According with the results of the antibiogram, we 


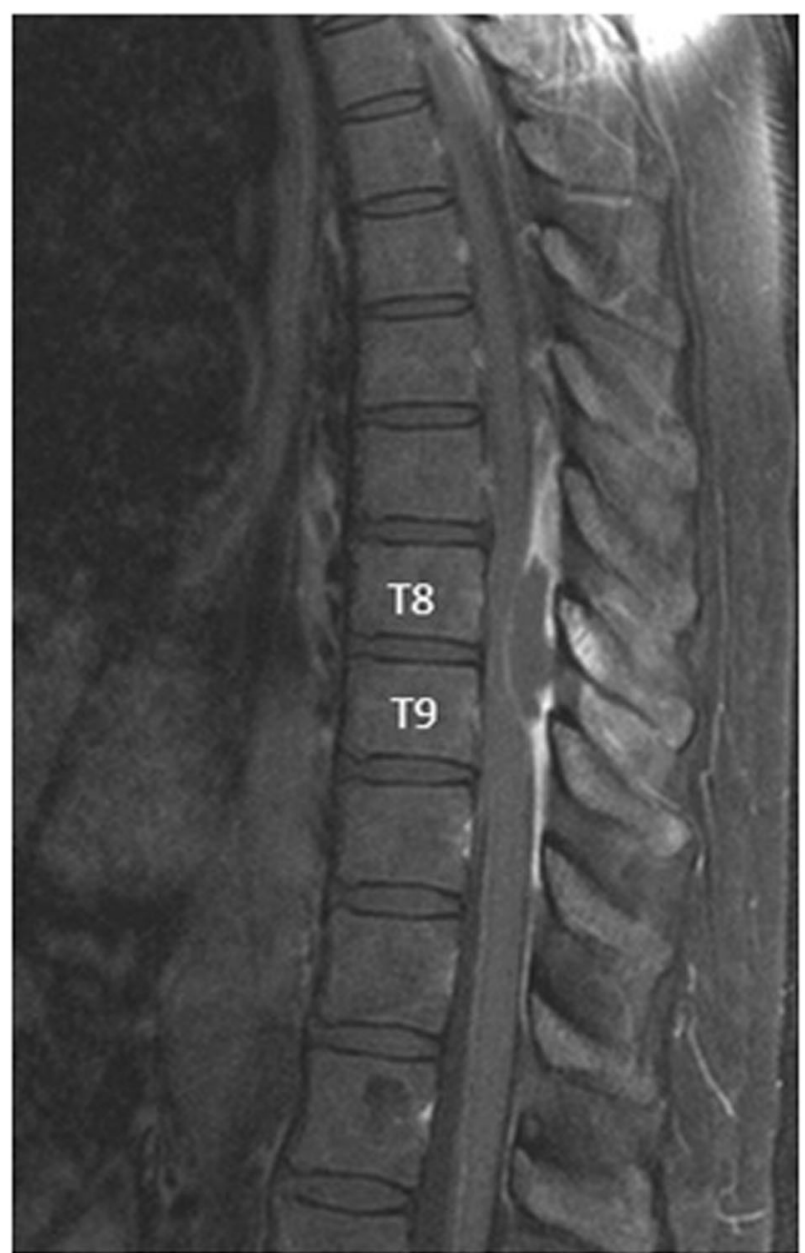

Fig. 3 Dorsal MRI shows an epidural abscess $(34 \times 10 \times 11 \mathrm{~mm})$. T1 sequence with dural hipercaptation

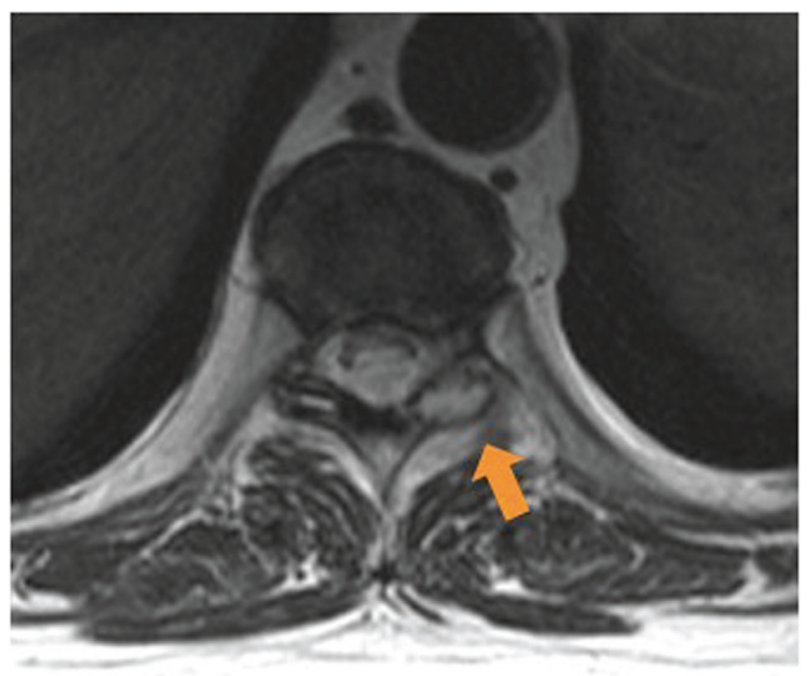

Fig. 4 Images from dorsal MRI shows articular liquid and bone oedema in the left facet joint at T8-T9 vertebrae. The narrow reflects the left facet joint

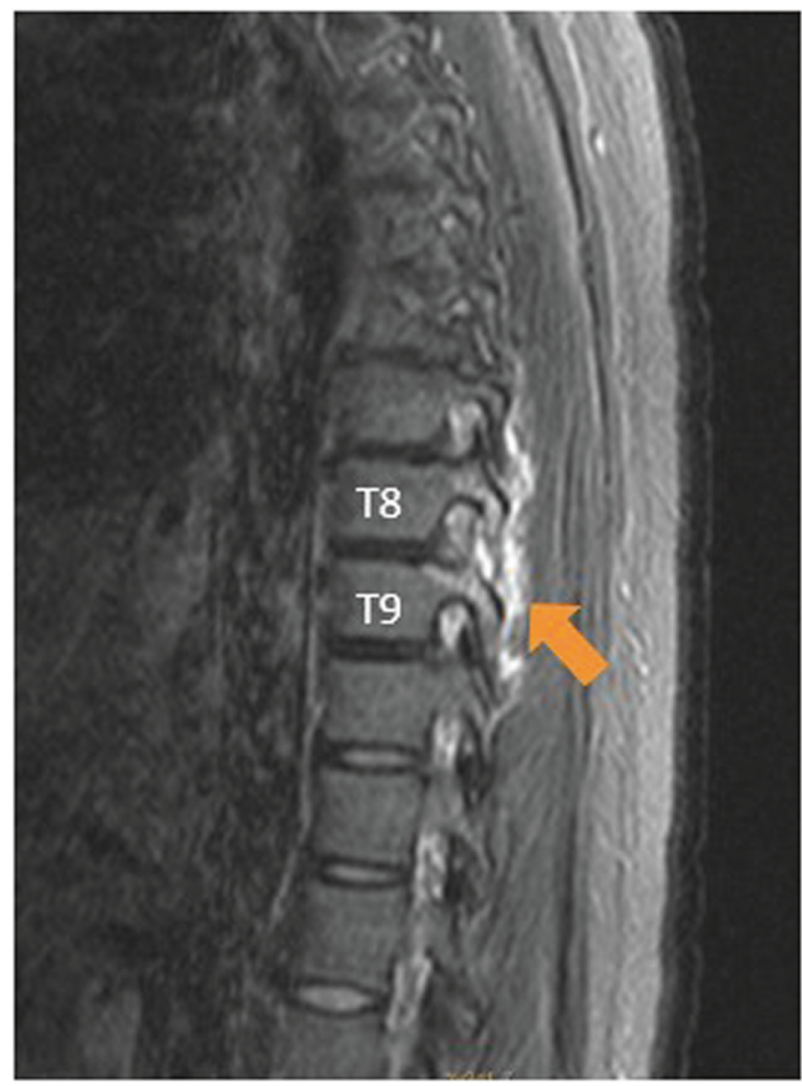

Fig. 5 Images from dorsal MRI shows articular liquid and bone oedema in the left facet joint at T8-T9 vertebrae. The narrow reflects the left facet joint

changed empiric antibiotic treatment to Levofloxacin $750 \mathrm{mg} / 24 \mathrm{~h}$ and completed treatment for 6 weeks.

In order to rule out possible sources of haematogenous seeding, a urine sediment and an echocardiogram were performed during hospital stay. These tests showed no alterations.

Inflammatory markers decreased in serial blood tests. Patient's evolution was satisfactory, and the motor strength of the left inferior limb improved to 4/5. Ambulation with a walker was tolerated.

Fourteen days after the surgery, the patient was discharged with physical therapy at home.

At 9 months follow-up, the MRI showed post-operative changes, mild myelopathy sequel at $\mathrm{T} 9$ but no residual collections or cavities (Figs. 6, 7 and 8).

After 1 year and a half from the surgery, the inflammatory markers (CRP and ESR) were normal. The patient has full recovery from the neurological deficit and mild residual back pain due to muscular insufficiency. Only a cane is needed for long distance ambulation. 


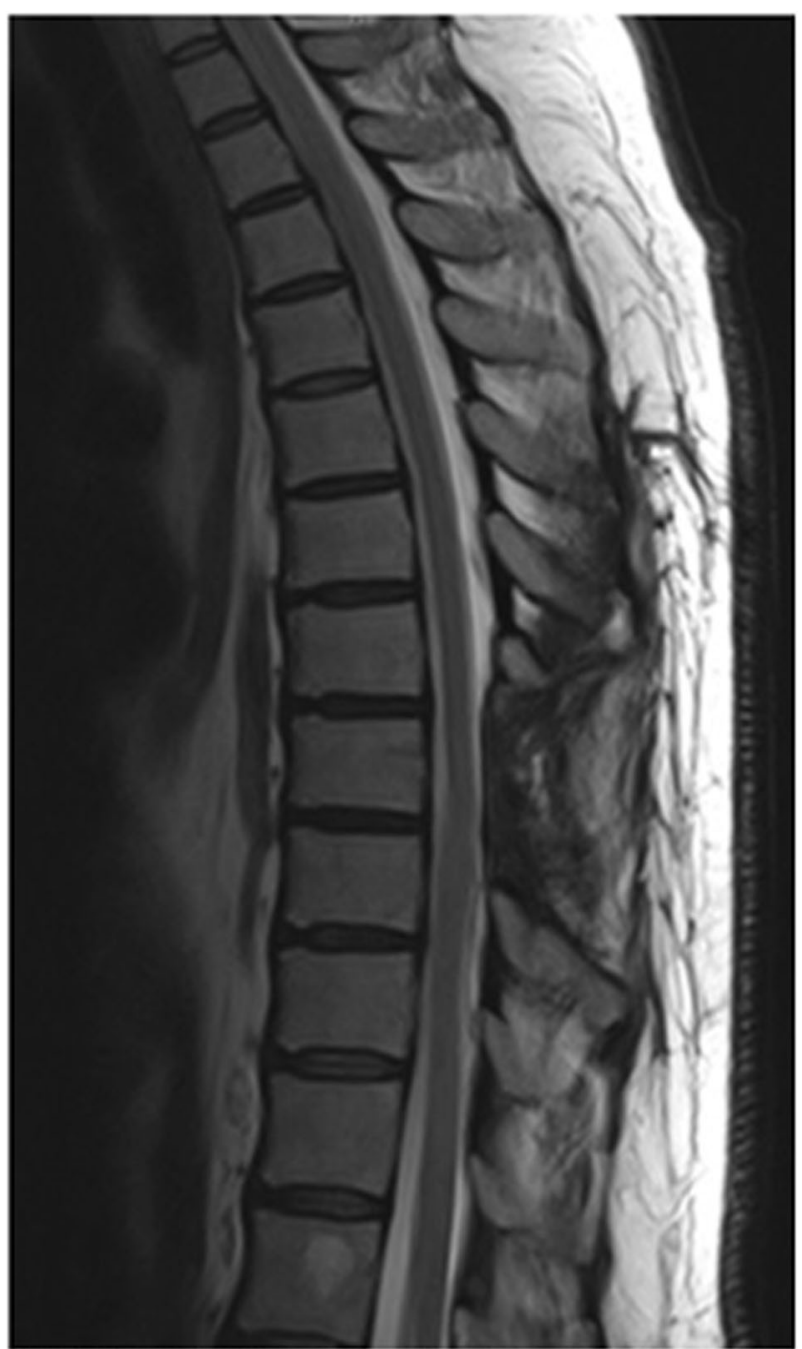

Fig. 6 MRI after 9 months of surgery. Residual mild myelopathy is observed

\section{Discussion}

SAFJ is an uncommon clinical entity that is often underdiagnosed. To the best of our knowledge, only a few articles have been published. High index of clinical suspicion is needed to diagnose this entity at the earliest possible stage.

Clinical signs and symptoms can vary between patients, and may resemble other pathologies such as spondylodiscitis $[3,5,8,9]$ or pyelonephritis [6, 8]. SAFJ generally presents as a unilateral back pain with often radiation to the flank or buttocks $[2,3,6,8]$. SAFJ seems to present with more acute and severe symptoms in early stages than spondylodiscitis [3].

Severe neurological deficits, as in our case, are present in $<10 \%$ of cases according to Narváez et al. [3]. Also, according to the same article, fever occurs in $83 \%$ of the cases.

The most frequent localisation is the lumbar spine $(86-97 \%)[3,5,6]$. In the case that we presented, the SAFJ

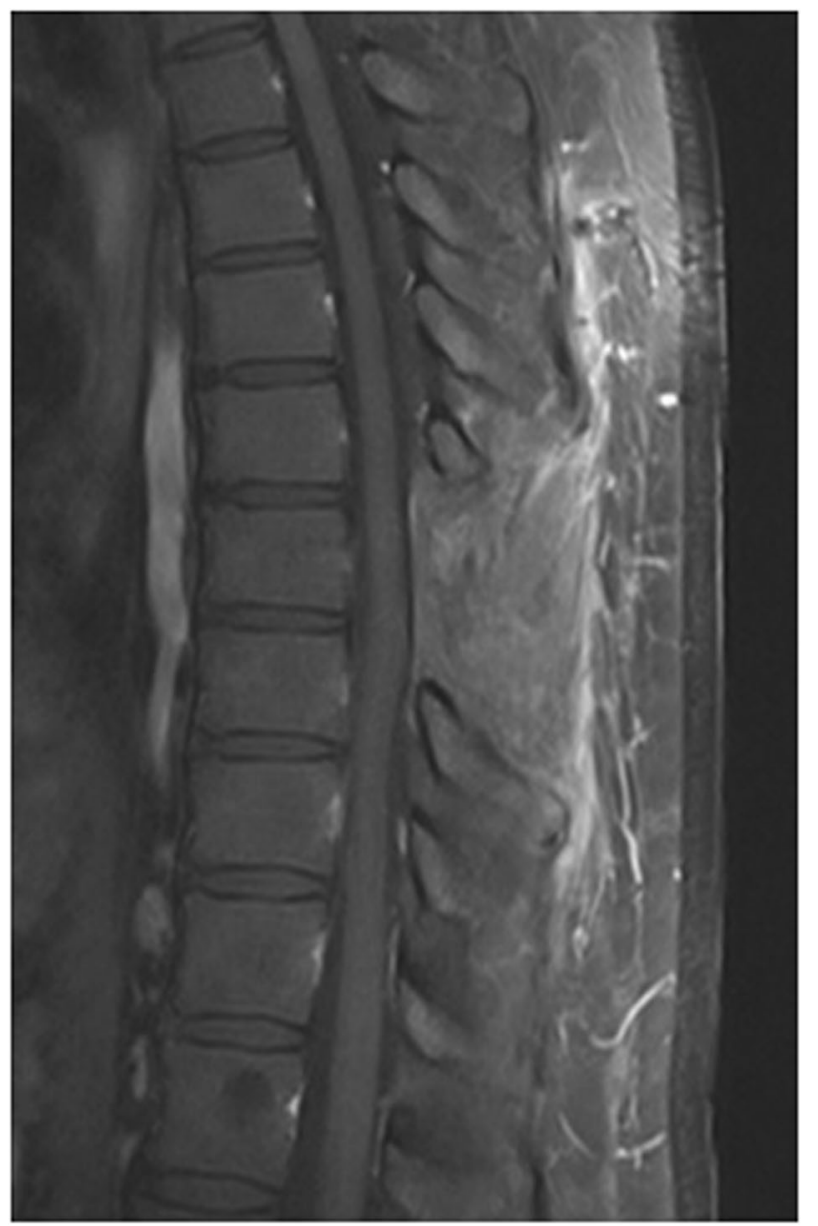

Fig. 7 MRI after 9 months of surgery. Residual mild myelopathy is observed

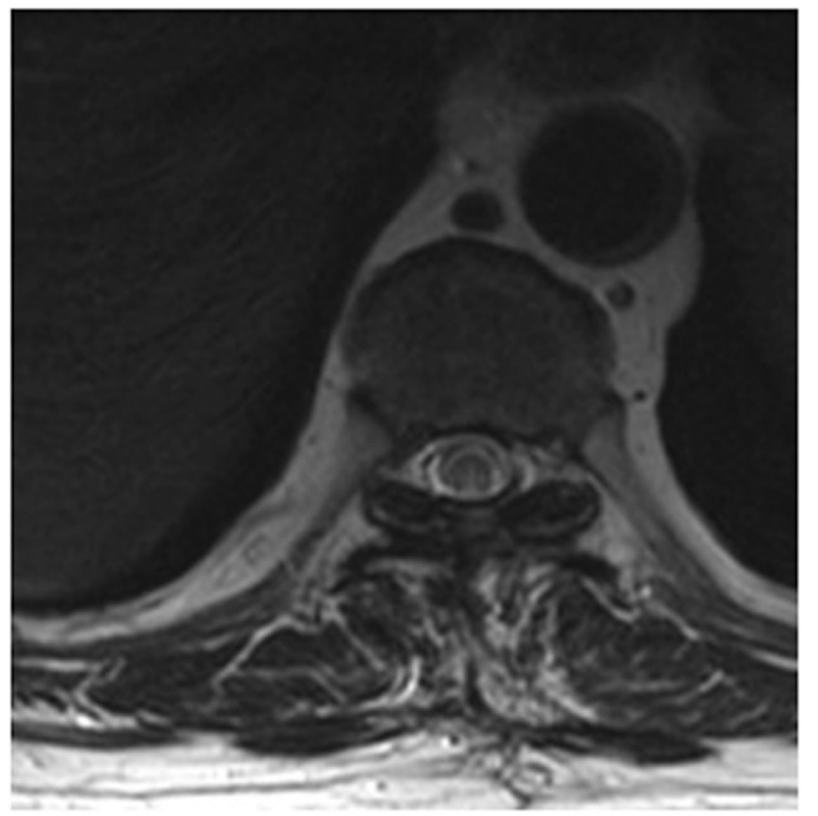

Fig. 8 MRI after 9 months of surgery. Residual mild myelopathy is observed 


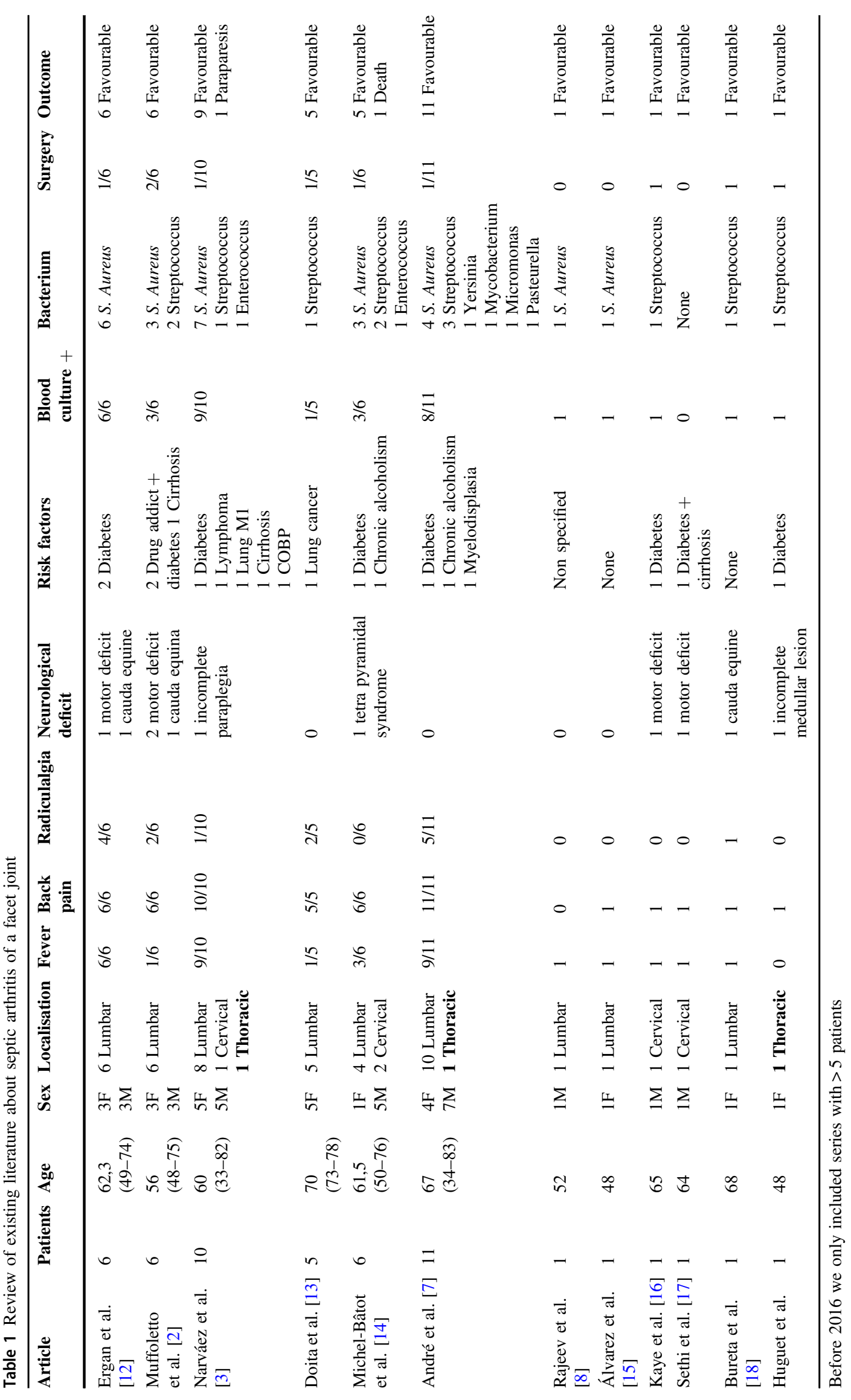


was localised in the left T8-T9 facet joint. This thoracic localisation is very unusual for SAFJ ( $4.5 \%$ incidence according to Narváez et al. [3]). There are only three more cases described in the literature with SAFJ in the thoracic spine $[3,7,10]$.

Diabetes mellitus and other immunosuppressive diseases such as chronic liver disease, alcoholism or corticosteroid therapy have been described as risk factors to develop SAFJ $[2,3,6-8]$. There have been cases of iatrogenic infection after invasive procedures like epidural injection, acupuncture or spinal surgery $[3-6,11]$.

However, very few cases have been described without any immunosuppressive condition or history of invasive procedures. $[5,6,15,18]$.

Age is another risk factor described in some publications, SAFJ seems to be more frequent in patient above 60 years old $[3,5,6]$.

Diabetes mellitus was the only risk factor present for our patient.

MRI remains as the gold standard for image diagnose [28]. Plain radiographs are not useful for diagnosis of SAFJ in early stages because radiologic abnormalities appear 6 to 12 weeks after the onset of symptoms [1-3, 5, 6]. Performing a CT scan is advantageous in order to guide percutaneous biopsies of the paravertebral abscesses or of the concerned facet joint $[3,6]$.

It is mandatory to identify the microorganism that causes the SAFJ. In cases where blood culture results are negative, an aspiration of the articular liquid of the facet joint needs to be performed $[3,6,8]$.

Since the most common cause of SAJF is haematogenous spread [2, 4, 6-8], it is important to search for any possible pathogen entry portals, although they remain unknown in one third of the patients [4]. Blood cultures must be done for all cases with suspicion of SAFJ before initiating any antibiotic treatment. Also, urine culture and echocardiogram (like we did in our case) could be useful to rule out a concomitant infectious process such as urinary tract infection or endocarditis [3].

In our case, at first, we could not find any entry portal that could explain the origin of the SAFJ. After re-interrogation, our patient mentioned dental manipulation weeks before the symptoms started that could be the entry portal that we were searching for.

Although, the most common pathogen described in the literature is S. aureus [2-9], we isolated a Streptococcus constellatus in our case. Streptococcus species are the 2nd most frequent pathogen described in SAFJ [2, 3, 7, 8]. Streptococcus constellatus is often associated with pharyngeal infections. This leads to think that the dental manipulation our patient had weeks before the onset symptoms could be the cause of her SAFJ.
Intravenous antibiotherapy remains as the gold standard $[4,7,8]$ for the treatment of SAFJ. It is difficult to determine the optimum duration of antibiotic treatment due to the heterogenicity of the cases $[3,9]$.

In presence of an abscess, unfavourable outcome despite antibiotic treatment or severe neurological symptoms, antibiotic treatment has to be associated with a surgical debridement [3-9]. As seen in our case, the patient presented a severe neurological deficit due to a medullar compression, so we decided to perform an urgent laminectomy to decompress the medullary canal.

In cases without neurological symptoms, percutaneous drainage under image guidance is not as invasive as a surgical procedure, and could provide a good outcome in most patients $[2,3,5]$.

As Narváez et al. [3] described in their publication a paraespinal/epidural extension does not indicate a worse prognosis. The overall prognosis for SAFJ is good with a mortality rate of $2 \%[3,7]$.

According to the literature, bed rest for 3 weeks is recommended [3, 7, 9].

With the correct treatment (according to the antibiogram) for the isolated microorganism, the symptoms should improve, and full recovery is expected [3, 7].

A review of existing literature is summarised in Table 1.

To conclude, SAFJ is a rare entity with a low incidence, and often underdiagnosed. As a result of misdiagnosed cases, aging and an increase in number of immunosuppressed patients, the incidence of SAFJ is increasing over the years $[3,5]$.

Physicians should have in mind and rule out this clinical entity to avoid the severe consequences that could develop an undiagnosed SAFJ.

Medical treatment with intravenous antibiotic therapy is indicated in non-complicated cases (if an abscess or neurological symptomatology are not present). Associating a percutaneous drainage of the abscess or joint implies a higher rate of success, as seen in some published articles [3].

In most cases, a full recovery is expected after an early diagnosis and specific treatment.

\section{Compliance with ethical standards}

Conflict of interest The authors declare that they have no conflict of interest.

\section{References}

1. Halpin DS, Gibson RD. Septic arthritis of a lumbar facet joint. $\mathbf{J}$ Bone Jt Surg. 1987;69B:457-9.

2. Muffoletto AJ, Ketonen LM, Mader JT, Crow WN, Hadjipavlou AG. Hematogenous pyogenic facet joint infection. Spine. 2001;26:570-1576. 
3. Narváez J, Nolla JM, Narváez JA, Martinez-Carnicero L, De Lama E, Gómez-Vaquero C, et al. Spontaneous pyogenic facet joint infection. Semin Arthritis Rheum. 2006;35:272-83.

4. Rombauts PA, Linden PM, Buyse AJ, Snoecx MP, Lysens RJ, Gryspeerdt SS. Septic arthritis of a lumbar facet joint cause by Staphylococcus aureus. Spine. 2000;25:1736-8.

5. Harries LK, Watura R. Septic arthritis of unilateral lumbar facet joint with contiguous abscess, without prior intervention. BMJ Case Rep. 2012. https://doi.org/10.1136/bcr.09.2011.4849

6. Kitova-John MB, Azim-Araghi A, Sheikh FT, Kitov BD. Idiopathic septic arthritis of a lumbar facet joint associated with paraspinal abscess. BMJ Case Rep. 2015. https://doi.org/10.1136/ bcr-2015-211135

7. André V, Pot-Vaucel M, Cozic C, Visée E, Morrier M, Varin S, et al. Septic arthritis of the facet joint. Med Mal Infect. 2015;45:215-21.

8. Rajeev A, Choudhry N, Shaikh M, Newby M. Lumbar facet joint septic arthritis presenting atypical as acute abdomen - a case report and review of the literature. Int $\mathbf{J}$ Surg Case Rep. 2016;25:243-5.

9. Krishnan V, Amritanand R, Sundaraj GD. Methicillin-resistant Staphylococcus aureus as a cause of lumbar facet joint septic arthritis. J Bone Jt Surg Am. 2010;92:465-8.

10. Pilleul F, Garcia J. Septic arthritis of the spine facet joint: early positive diagnosis on magnetic resonance imaging. A review of two cases. Rev Rhum Fr. 2000;67:237-40.
11. Daivajna S, Jones A, O’Malley M, et al. Unilateral septic arthritis of a lumbar facet joint secondary to acupuncture treatment-a case report. Acupunct Med. 2004;22:152-5.

12. Ergan M, Macro M, Benhamou CL, Vandermarcq P, Colin T, L'Hirondel JL, et al. Septic arthritis of lumbar facet joints. A review of six cases. Rev Rhum Engl. 1997;64:386-95.

13. Doita M, Nabeshima Y, Nishida K, Fujioka H, Kurosaka M. Septic arthritis of lumbar facet joints without predisposing infection. J Spinal Disord Tech. 2007;20:290-5.

14. Michel-Batôt C, Dintinger H, Blum A, Olivier P, Laborde F, Bettembourg B, et al. A particular form of septic arthritis: septic arthritis of facet joint. Jt Bone Spine. 2008;75:78-83.

15. Álvarez J, Fernández F, González M, De la Fuente J. Artritis séptica facetaria con absceso epidural y paraespinal: a propósito de un caso. Enferm Infecc Y Microbiol clínica. 2016;34:76-7.

16. Kaye I, Protopsaltis T. Cervical facet joint infection and associated epidural abscess with Streptococcus intermedius from a dental infection origin: a case report and review. Bull NYU Hosp Jt Dis. 2016;74:237-43.

17. Sethi S, Vithayathil MK. Cervical facet joint septic arthritis: a real pain in the neck. BMJ Case Rep. 2017. https://doi.org/10.1136/ bcr-2016-218510

18. Bureta C, Tominaga H, Yamamoto T, Setoguchi T, Kawamura H, Nagano S, et al. Lumbar spine epidural abscess and facet joint septic arthritis due to Streptococcus agalactiae: a case report. BMC Surg. 2018;18:16. 